\title{
Planetary Protection Knowledge Gaps and Enabling Science for Human Mars Missions
}

\author{
J Andy Spry (SETI Institute) 818-414-2471, aspry@ seti.org
}

July 15,2020

Co-authors/reviewers with respective institutions:

Bette Siegel (NASA HQ); Gerhard Kminek (ESA); Corien Bakermans (Penn State Univ);

J Nick Benardini (JPL/Caltech); Esther Beltran (Univ Central Florida); Rosalba Bonaccorsi (SETI Institute);

John Canham (Northrop Grumman); Marie-Christine Desjean (CNES France)

Peter Doran (Louisiana State Univ); John E Hallsworth (Queen's Univ Belfast);

Javier Martin-Torres (Univ Aberdeen); Jill Mikucki (Univ. Tennessee); Tullis Onstott (Princeton);

George Profitiliotis (National Technical Univ of Athens); Aaron Regberg (NASA JSC);

Petra Rettberg (DLR Cologne); John Rummel (Friday Harbor Partners LLC); Kevin Sato (NASA Ames);

Andrew Schuerger (Univ Florida); Nitin Singh (JPL/Caltech); Carol Stoker (NASA Ames);

Kasthuri Venkateswaran (JPL/Caltech); Robert Zimmerman (Symbiotek Systems);

Maria-Paz Zorzano (INTA Spain)

Cosigners (21 at the time of submittal) can add their endorsements of this White Paper at:

https://docs.google.com/spreadsheets/d/114XXZSXWHScjy3lygmwXPGlbFbf1S2tLNetoS_wE76Y/ edit\#gid=0 


\section{Rationale}

In the coming decade, as we prepare for the first mission to Mars with a human crew, we have a continuing obligation to protect the integrity of scientific investigations at Mars. In particular, it is unlikely that the search for life on Mars will be completed by the time the first crew systems arrive at the martian surface. Indeed, some consider the presence of astronauts to be an essential augmentation to the robotic search for evidence of life on the red planet.

In addition, the environment of the Earth needs to be protected from the threat posed by the uncontrolled release of a putative martian life form into the terrestrial biosphere. Prevention of such harmful crosscontaminations between Mars and the Earth is the practice of planetary protection. At present, the knowledge of how to achieve these two goals (prevention of forward contamination from Earth and backward contamination from Mars) is well described for robotic systems. In contrast, for human missions there are only guidelines (Kminek et al. 2017a) but no engineering requirements, in part, because our knowledge of Mars (and of how contamination from crewed systems will interact with Mars) is incomplete. These gaps in our knowledge (as also described in NASA NID8715.129) need to be addressed through acquisition of new data during the next decade, if planetary protection measures are to be implemented successfully for human missions. This paper describes the technical issues and measurements needed.

\section{Approach}

Since 2015, the topic of planetary protection knowledge gaps (KGs) for human missions has been systematically addressed during a series of workshops (e.g., Race et al., 2019), currently being held under the COSPAR banner. The international multidisciplinary group of scientists and engineers involved in this activity have generated and refined a set of KGs, and grouped them into three study areas: 1) natural transport of contamination on Mars; 2) microbial and human health monitoring, and; 3) technology and operations for contamination control. Closure of these KGs would lead to an end-to-end knowledge-based solution for setting planetary protection requirements and developing implementation procedures for human missions. The group has performed assessments of the measurements and data needed to close each of the KGs, together with an identification of locations/destinations and instruments needed for making those measurements.

\section{Planetary Protection Knowledge Gaps}

\subsection{Natural Transport of Contamination on Mars}

\subsubsection{Surface/Atmospheric Transport}

The aerial transportation of terrestrial organisms in the martian atmosphere could bring them to a "Special Region"1 (Rummel et al., 2014) even if a crewed spacecraft lands at a distant "non-special" location. Understanding the possibility of aerial transportation of terrestrial organisms on Mars is therefore of paramount concern. Although atmospheric modeling implies the consideration of a number of uncertainties, we have a good knowledge of atmospheric dynamics on Mars, and low-fidelity model estimates can be generated for the general case at a coarse scale.

The surface observations of the Mars Science Laboratory Curiosity rover, show that the typical airborne dust aerosol size on Mars is of the order of $1-2 \mu \mathrm{m}$ in radius, except during the onset of global dust storms, when dust radii quickly exceeded $3 \mu \mathrm{m}$, reaching maximal sizes of $8 \mu \mathrm{m}$ effective radius (Lemmon et al., 2019). During such a global storm, the typical aerosol size in suspension can be greater than $3 \mu \mathrm{m}$ for around 60 martian sols, while the atmosphere clears by deposition of the larger particles and only the

\footnotetext{
${ }^{1}$ A Special Region is defined as a naturally occurring environment on Mars that is considered habitable for Earth microorganisms based on temperature $\left(>-28^{\circ} \mathrm{C}\right)$ and water activity $(>0.5)$ parameters.
} 
smallest dust remains in suspension. If individual spores are lifted by winds and transported through the atmosphere, they will be exposed to a high UV fluence during the time of flight. A layer of $8 \mu \mathrm{m}$ of mineral dust can screen only $20 \%$ of the incident UV radiation (Muñoz Caro et al., 2006), however the opacity of the atmosphere during such a storm is so reduced that the UV fluence can decline by a factor of $\sim 1000$. A credible scenario from a planetary protection point of view could be an event when winds are strong enough to lift-off larger sized particles, including viable microorganisms protected by dust grains and/or as part of a microbial community or biofilm fragment. During this scenario, incident UV radiation would be screened during the dispersal, before settling elsewhere on the planet.

At the present time, knowledge of where and how far such particles would travel, particularly on the mesoscale of interest relative to human exploration, is absent. The finding of the 2018 COSPAR working meeting on this topic (Race et al. 2019) is that measurements have not been taken at the necessary frequency and duration to create predictive models with a reasonable level of confidence. The experts at this meeting recommended dedicated meteorology investigations in the area of the planned human landing site, to take new measurements over at least a full martian year at multiple fixed locations to obtain the data needed to fully develop, test and validate contamination transport models. However, it was observed that a single suitably accommodated high-fidelity meteorology package on a mission could enable the accumulation of sufficient data, but would produce a less robust model. The 2018 COSPAR group considered that it would be prudent for meteorological measurements to be given high priority in future human and robotic exploration of Mars.

\subsubsection{Subsurface Transport}

Subsurface transport can be considered on multiple scales from shallow (0-2.5 meters) to km depths, and transport of contaminants are different for each. Shallow drilling such as the proposed Icebreaker mission (McKay et al. 2013) could collect ice-saturated samples at 0-1 meters depth and assess their habitability and assay for martian life biosignatures protected from surface chemistries. This near surface environment is at greatest risk from contamination from human activities, but is of the most interest in the near term for reasons of accessibility and potential use as a resource. The ice table beneath $10 \mathrm{~s}$ of $\mathrm{cm}$ of soil could host perchlorate brines but they are not expected to be habitable at modeled temperatures (Rivera-Valentin et al. 2020). But, implicit in the "Special Regions" concept is that habitable environments can occur on Mars, and therefore martian life in such environments can't yet be ruled out. Life detection investigations of sites that may host Mars life would be an important input for future human missions (Stoker et al. 2020). At moderate depths (up to 100s meters) subsurface temperatures should block penetration of fluid borne physical/chemical/biological contaminants by freezing. As long as these contaminants are not themselves generating heat, then the underlying potential martian aquifer where fluid transport could transmit contamination on a regional scale will not be accessed. Transport of contaminating terrestrial organisms from surface operations into a deep subsurface aquifer, could result in alteration or destruction of a hypothetical martian ecosystem. However, there is currently no identified mechanism for direct contaminant transport to these deep environments.

Access to caverns represents a more challenging exploration environment. It is conceivable that because of high diurnal surface temperature variations that aerial inhalation/exhalation exists. If so, even activities proximal to the cavern entrance could disperse terrestrial contaminants widely within a cave system, and potentially also from further afield, given the atmospheric transport threat described in 3.1.1. On Earth caves into permafrost typically seal themselves from the atmosphere with ice at their coldest point so it seems unlikely that caves will openly penetrate to the sub-permafrost aquifer layer. However, martian 
caves could in their upper reaches potentially possess enough liquid water to support local brine habitats of astrobiological interest and would be vulnerable to contamination.

\subsubsection{Lethality of the Mars Environment to Terrestrial Contaminant Organisms}

Numerous biocidal factors persist in the martian surface, shallow subsurface, and aeolian environments. Some biocidal factors are widespread on Mars (e.g., UV irradiation), while other factors might be spatially or temporally constrained (e.g., heavy metals or high salts in diverse regoliths). The top 7 most biocidal factors for inactivation of Earth microbes arriving on spacecraft have been described as follows: (1) solar UVC and UVB radiation, (2) extreme desiccation (i.e., low $\mathrm{a}_{\mathrm{w}}$ ), (3) solar UV-induced volatile oxidants (e.g., O2-, O-, $\mathrm{H} 2 \mathrm{O} 2, \mathrm{O} 3$ ), (4) high salt concentrations in some regolith (e.g., $\mathrm{MgCl}$, $\mathrm{NaCl}, \mathrm{FeSO} 4$, and MgSO4), (5) acidic conditions in many regoliths, (6) solar particle events, and (7) low-pressure (2-12 mbar). (Hallsworth et al., 2007; de Vera et al., 2010; Rummel et al., 2014; Schuerger et al., 2012; 2013). Synergistic effects of different environmental parameters are an important consideration because, on Earth, conditions that are biocidal to most organisms may be preferred environments for niche extremophile organisms. Few studies have looked at synergistic effects of the parameters listed above on microbial survival, metabolism, growth, and adaptation (Nicholson et al., 2010; Wadsworth and Cockell, 2017). Furthermore, there is evidence that substances considered inhibitory or biocidal on Earth can actually favor microbial growth at low temperatures that are within the range experienced on Mars (Chin et al., 2010). Thus, a key goal of future research should be the development of experiments and models to understand multi-factorial combinations of biocidal or inhibitory factors under conditions relevant to Mars. Such experiments need to consider: 1) the limiting (individual and combined) effects of (for example) UV, desiccation, oxidants, regolith and aerosol geochemistry, low pressure, temperature, and gas composition on microbial survival; 2) the effects of biocidal factors on the survival of cultivable and non-cultivable bacteria, archaea, fungi, and other eukarya present on spacecraft; 3 ) the lethality kinetics of (1) and (2) relative to exposure and time; and 4) the effects of air column composition and opacity, spores within aerosols, and spacecraft surfaces on kill-curve kinetics in order to develop a quantitative model(s) of microbial survival on Mars for robotic and human surface operations. Furthermore, a fundamental understanding of the mechanisms of lethality would allow models to apply effects of exposure (with margins) to any microbe (as well as to the degradation of organic matter more generally). Following similar considerations, models for microbial growth and adaptation (as opposed to just survival) under conditions found in Special Regions should also be developed. Key to closing this KG is the validation of models of microbial survival and growth on Mars with flight experiments on Mars and precursor missions with appropriate ground testing.

In considering human missions to Mars, models and experiments need to factor in additional conditions that will act to buffer or protect Earth microorganisms from biocidal factors on Mars. These conditions include: dust loading on spacecraft surfaces (Schuerger et al., 2012); terrain and/or spacecraft shadowing that decreases UV irradiation and wind dispersal of microbes from spacecraft surfaces (Moores et al., 2007); the formation, transport, and shielding efficiency of particulates released from habitats; structural architecture/ site arrangement of landed elements (e.g., increased humidity under large structures); and the presence of biofilms and other cell debris around attached terrestrial organisms (Billi et al., 2019). As little as $0.5 \mathrm{~mm}$ of martian fines can attenuate the biocidal effects of UV irradiation by a factor of $1 \times 10^{5}$ (Mancinelli and Klovstad, 2000). When shielded from UV, oxidative effects in the surface and shallow subsurface environment are predicted to have significant biocidal effects; however, these effects are still not well-constrained for the martian surface (Kminek et al. 2017b).

\subsection{Microbial and Human Health Monitoring (MHHM)}




\subsubsection{Microbial Monitoring of the Spacecraft Environment}

New advancements in genomics and DNA sequencing technology have deepened our understanding of microbial populations and their interactions to the point where diagnostic monitoring of the microbiome of the built environment is achievable (e.g. Lax et al., 2014). Likewise, these advancements have been applied to isolate and characterize microbes from spacecraft-associated environments (e.g., Singh et al., 2019), and provide the potential for a data-rich cost- and mass-effective tool for long term monitoring of the health and functional performance of the spacecraft environment and its systems, as well as the threat posed to Mars by the spacecraft microbiome.

The ability to sample for genomic data broadly, then use software tools to focus on relevant microorganisms, is key to the unique utility of the method. Using metagenomics data generated from a spacecraft assembly facility and the International Space Station (ISS), environmental samples were analyzed for radiation-resistant non-spore forming microorganisms (Be et al. 2017). Among the wide spectrum of DNA sequence reads associated with microorganisms ( $\sim 7$ million), only 600 to 6,000 reads $(\sim 1$ to $10 \%$ ) were related to the functional gene sequences of the radiation resistant microorganisms of interest. Focused development of spacecraft specific nucleic acid databases to assist in future instrumentation/ experiment development, identifying and characterizing core microbiomes, assessing microbial changes to the environment and determining the prevalence of relevant microorganisms is necessary for this technology to be fully exploited as a risk analysis tool.

\subsubsection{Microbial Monitoring of Crew Health}

A critical component of a crewed mission to the surface of Mars is monitoring astronaut responses to exposure to the martian environment, particularly if the presence of a martian biosphere is suspected or detected. Evaluation of human health requires the monitoring of the crew themselves, as well as all the microbial communities associated with spacecraft systems described in 3.2.1 previously. These microbial florae need to be monitored and tracked not only at their initial state against an established baseline, but also for changes over time.

Currently, microbial monitoring of crew health during human missions is culture-dependent and reliant on ground-based analysis. Both of these features are problematic for longer duration missions that leave LEO (low earth orbit). Culturing microbes requires a significant amount of materials and supplies that may not fit within the strict mass requirements of early crewed missions. Additionally, culture-based methods that are currently sufficient for monitoring crew health in LEO are likely insufficient for identifying novel or fastidious organisms that could pose a health hazard. Culture independent methods like DNA sequencing generate large amounts of data ( $\mathrm{Gb}$ to $\mathrm{Tb}$ ) that can provide the necessary breadth and depth to monitor the crew microbiome and can be processed and analyzed locally. NASA is pursuing DNA-sequencing methods for microbial monitoring based around the MinION platform. While sample preparation aboard the ISS has been demonstrated, significant optimization will be required to support extended mission duration.

The 2018 COSPAR workshop (Race et al. 2019) identified crew health microbial monitoring as a significant KG that could be addressed by developing and implementing a routine monitoring plan for all current and future missions. Samples should be collected at a higher frequency than at the (present) quarterly level, and be archived.

A human health baseline must be established so that deviations can be detected. Crew health should be framed in context of event detection and risk, identifying critical steps and issues that pose risks; categorize those risks, identify by their likelihood and consequence to create a stepped approach to operational decision-making. DNA sequencing can be used to collect the needed data and integrate it with other data sets to understand the true key health issues, including prioritization of contamination risk, assessment of 
the best protocols for control and mitigation (see 3.2.3 below), and a risk-based assessment for crew health maintenance. Additionally, ecological changes in the built environment may be leading indicators for the introduction of new organisms that could affect crew health. In order to facilitate this monitoring, an autonomous or semi-autonomous orthogonal technique such as near infrared spectroscopy, proteomic, Raman or antibody-based approaches should be developed in parallel with sequencing based approaches.

\subsubsection{Mitigation of Microbial Growth}

NASA and other space agencies have mitigated biological contamination of spacecraft destined for certain solar system targets from the 1960's to the present day. These include the most recent Europa Clipper, Mars 2020 and ExoMars missions. For such robotic spacecraft, the key tenets for microbial growth mitigation consist of appropriate design, microbial reduction, cleanliness verification loops and recontamination prevention, implemented throughout the hardware life cycle (Benardini et al. 2014). In contrast, human spacecraft systems are not designed to be "sterilizable", nor should they be, since the presence of the crew means that the substantial microbial community (microbiome) that is travelling with the humans in the spacecraft would almost immediately begin to recontaminate the sterilized environment. Wherever humans have unmitigated direct contact with an environment, their microbes are transferred, so mitigations will be required if microbial release into the martian environment is to be controlled. Although there have been mission-enabling technology advancements for understanding both cleanroom microbial contamination and microbial reduction parameters (e.g. heat and vapor hydrogen peroxide Chung et al. 2006, Schubert et al. 2011), microbial mitigations for crewed missions have an increased level of complexity. Microbial surveillance and tracking studies on the ISS have demonstrated that the microbiology of its built environment changes to include the microbes of each successive crew who lives on the ISS (Voorhies et al 2019, Avila-Herrera et al 2020). Cleaning and sterilization methods only temporarily reduce the microbial load on a surface, which becomes re-populated with (different) microbes over time. This necessitates a tolerance of recleaning approaches.

Spacecraft microbial reduction protocols can be leveraged from earlier NASA robotic missions, separately or in combination, with additional operational cleaning strategies such as anti-microbial wipes, vaporized sterilizing agents, and germicidal lighting. Autoclave or other gaseous methods may be considered for small tools and equipment. Additionally, surfaces impregnated with anti-microbial agents or naturally antimicrobial materials could be used (Sobisch et al, 2019). However, some elements of a habitat or capsule may be incompatible with anti-microbial surface treatments, anti-microbial material choices or aggressive cleaning processes, so end-to-end strategies need to be developed.

Microbial mitigations need also to be built into operational concepts. Current exploration concepts feature an airlock facility, which houses the pressurized suits and equipment for martian surface exploration. That could be isolated and separately sterilized prior to astronaut exit to the martian surface, including exterior surface sterilization of the pressurized suits after the crew has suited up. Approaches would also be necessary for research tools and instruments, robotic support equipment and returned samples. However, it would be important to continue microbial monitoring protocols (3.2.1) to verify cleaning effectiveness, anti-microbial material effectiveness, predict cleaning timelines, and microbial adaptation or resistance (Sielaff et al. 2019). These microbial monitoring protocols would also help catalogue the type of microbial contamination that the martian environment may be exposed to, which is similar to that of the characterization being conducted on the Mars Sample Return Campaign (Farley et. al 2017). Testing of hardware and protocols would need to be conducted using ISS and/or terrestrial analog closed system habitat facilities, to determine the most efficient (and sufficient) habitat/operations mitigation approaches 
and protocols to allow effective crewed exploration on the martian surface while mitigating both forward and backward contamination risk.

\subsection{Technology and Operations for Contamination Control}

Critical to the success of planetary protection implementation for human missions is integration of the transport and survival characteristics of terrestrial microorganisms at Mars, as well as the capability to monitor the nominal (and detect non-nominal) microbiome profile of the crew and human systems, into hardware technology and operations. Such integration needs to happen in parallel with development of mission architecture and design concepts in the course of the 2020s, in order to be incorporated into engineering designs and spacecraft hardware in the 2030s.

The COSPAR meeting series has identified a series of contamination control KGs related to technology and operations of crewed systems, based on then current mission concepts (NASA 2015). Independent of a specific mission concept, these are the general topic areas of: knowledge of Mars and permitted contamination levels versus hardware operational performance; approaches to astronaut health and preventing back contamination, and; development of policy to address these first two topic areas. At the present time, having adopted many of the findings of the COSPAR workshop series into an Interim Directive (NID8715.129; NASA, 2020), NASA is beginning the process of incorporating these KGs into hardware design roadmaps, so that design and performance decisions for contamination control can be matured in parallel with engineering designs. However, in order to reduce design conservatism (which may increase mass and cost) resulting from accommodating design uncertainty in planetary protection requirements, the KGs in transport and survival, as well as microbial monitoring, need to be addressed. It is critical that requirements for biological and non-biological contaminants be validated and verifiable.

The best approach for addressing contamination issues with regard to human exploration of Mars is to utilize knowledge from addressing molecular, particulate and microbial contamination from missions in the 2020s, then tailoring the measurements for future human Mars exploration as necessary. Field technology trials with humans involved at different capacities (Cockell et al., 2018), in terrestrial or lunar settings represent a viable arena for missions involving astronauts training for Mars exploration, and they can specifically address the KG of how contamination from crewed systems will interact with Mars.

\section{Discussion and Conclusions}

Robotic missions to Mars have revealed a diverse, active geology, but not all places on Mars are equal in terms of providing the right conditions for microbial growth, resulting in the concept of "Special Regions" which was introduced into the COSPAR Planetary Protection Policy (Kminek et al., 2017a). In our present state of knowledge, this recognition of differential need of protection will likely continue into the human exploration era. Enabling human missions to Mars without compromising the safety of the Earth and the continued scientific exploration of Mars requires an informed partitioning of the martian surface, establishing quantitative planetary protection requirements and developing associated mitigation measures. The high-priority KG areas that need to be closed before we can actually do this are in understanding of:

- Source terms - biological contamination of a crewed system

- Distribution terms - dispersal of biological contamination released into or from the martian environment

- Reduction terms - biocidal and inhibitory effects of the martian environment on the biological contamination.

Addressing these KGs requires NASA and other Space Agencies to engage in the following activities:

- More frequent and systematic microbial monitoring of ISS crews and associated ISS environments to obtain statistically relevant data over long time periods and multiple crew complements. Such 
systematic microbial monitoring would need to be extended to cis-lunar and lunar environments, i.e. beyond the protective terrestrial magnetic field, in the 2020s.

- High-frequency, long-duration and multiple-location meteorological investigations on the martian surface. Such robotic precursor missions to Mars would cover two aspects at once: enabling scientific measurements in preparation for human missions to Mars in line with MEPAG goal IV (prepare for human exploration) and an additional opportunity for scientific investigations in line with MEPAG goal II (understanding the processes and history of climate on Mars) (MEPAG 2020).

- Ground-based testing; to model multi-factorial combinations of biocidal/ inhibitory factors on biological contamination, and; to demonstrate with humans the needed operational concepts for use at Mars. As when humankind began to explore our own planet, human missions to Mars can bring great benefits, but also risks, not only to the explorers and adventurers that go on these voyages, but for all of us and the generations to come. Knowledge-based decisions before engaging in such an enterprise are therefore important, not only to manage the resources deployed to achieve human presence on Mars, but also as a responsible way to control the risk.

\section{Summary}

In order to preserve the integrity of science at Mars and to assure the safety of both returning astronauts and the terrestrial biosphere during crewed exploration, progress must be made in the next decade to obtain the scientific data for timely closure of KGs and the definition of appropriate planetary protection requirements for crewed missions to Mars.

Much of this essential data would result from measurements made by instruments accommodated on precursor martian missions, and on crewed missions on the ISS and in the cis-lunar and lunar surface environments. Of highest priority among these are measurements to generate predictive weather and contamination transport models, and to understand the subsurface ice-aqueous transition environment at sites close to or representative of the first human Mars mission.

\section{References}

Avila-Herrera, A. et al., (2020) PLOS ONE, 15(4) https://doi.org/10.1371/journal.pone.0231838

Be N.A. et al., (2017) Microbiome 5:81.

Benardini, J. N. et al., (2014) Astrobiology, 14:1, $27-32$.

Billi, D. et al., (2019) Astrobiology 19 1008-1017 http://doi.org/10.1089/ast.2018.1900

Chin et al., (2010) PNAS 107, 7835-7840

Chung et al., (2006) JPL Study Report on Hydrogen Peroxide

Cockell, C.S, et al., (2018) Int. J. Astrobiol, https://doi.org/10.1017/S1473550418000186

de Vera, J.-P. et al., (2010) Astrobiology 10, 215-227.

Farley K. et al., (2017) https://www.hou.usra.edu/meetings/lpsc2017/pdf/2251.pdf

Hallsworth et al. (2007) Environ Microbiol 9 (3), 801-813

Kminek et al., (2017a) Space Res Today 200 12-25

Kminek et al., (2017b) 1st (2016) COSPAR Workshop Report

Lax S, et al., (2014) Science 345:1048-52.

Lemmon, M. T. et al., (2019). Geophys Res Lett, 46 9448-9456 https://doi.org/10.1029/2019GL084407

Mancinelli, R.L.\& Klovstad, M (2000) Planet. Space Sci. 48, 1093-1097.

McKay, C.P. et al. (2013) Astrobiology 13(4), 334-353.

MEPAG (2020) https://mepag.jpl.nasa.gov/reports/MEPAGGoals_2020_MainText_Final.pdf

Moores, J.E. et al., (2007) Icarus v 192 pp.417-433.

Muñoz Caro, G. M. et al., (2006) Sensors (Basel, Switzerland) vol. 6,6 688-696 
NASA (2015) Journey to Mars

NASA (2020) https://nodis-dms.gsfc.nasa.gov/library/OPD docs/NID 8715129 .pdf

Nicholson, W. L. et al., (2010) Appl. Environ. Microbiol. 76, 7559-7565.

Race, M. S. et al., (2019) 2nd (2018) COSPAR Workshop Report

Rivera-Valentin, E.G. et al. (2020) Nature (Astronomy) Letters. https://doi.org/10.1038/s41550-020-1080-9

Rummel, J.D. et al., (2014) Astrobiology 14, 887-968.

Schubert WW \& Beaudet RA (2011) Astrobiology 11(3):213-223.

Schuerger, A. C. et al., (2012) Planetary Space Sci. 72, 91-101.

Schuerger, A. C. et al., (2013) Astrobiology 13, 115-131.

Sielaff, A.C. et al., (2019) Microbiome, 7:50. https://doi.org/10.1186/s40168-019-0666-x

Singh NK et al., (2019) Appl Microbiol Biotechnol doi:10.1007/s00253-019-09813-z.

Sobisch, Y-L. et al., (2019) Front. Microbiol, https://doi.org/10.3389/fmicb.2019.00543

Stoker, C.R. et al. (2020) We should search for extant life etc. White paper submitted to Decadal Survey.

Stoker, C.R. et al. (2010) J. Geophys Res. 115, E00E20.

Voorhies, A.A. et al., (2019) Sci Rep, 9(1):9911. doi: 10.1038/s41598-019-46303-8

Wadsworth, J. \& Cockell, C.S. (2017) Nature, Sci.Rep. 7, Art.4662 https://doi.org/10.1038/s41598-017-04910-3 